\title{
COCHLEAR APLASIA- A RARE CASE
}

\author{
Kajal Agrawal ${ }^{1}$, Rajesh Kumar Rathore2, Akshat Chauhan ${ }^{3}$, Rajesh Kumar ${ }^{4}$
}

${ }_{1}^{1}$ Postgraduate Student, Department of Radiodiagnosis, R. D. Gardi Medical College and C. R. Gardi Hospital, Ujjain (M.P.)

${ }^{2}$ Associate Professor, Department of Radiodiagnosis, R. D. Gardi Medical College and C. R. Gardi Hospital, Ujjain (M.P.)

${ }_{3}^{3}$ Postgraduate Student, Department of Radiodiagnosis, R. D. Gardi Medical College and C. R. Gardi Hospital, Ujjain (M.P.)

${ }_{4}^{4}$ Postgraduate Student, Department of Radiodiagnosis, R. D. Gardi Medical College and C. R. Gardi Hospital, Ujjain (M.P.)

HOW TO CITE THIS ARTICLE: Agrawal K, Rathore RK, Chauhan A, et al. Cochlear aplasia- a rare case. J. Evolution Med. Dent. Sci. 2017;6(95):7063-7065, DOI: $10.14260 /$ jemds/2017/1533

\section{PRESENTATION OF CASE}

To report a rare case of cochlear aplasia in a 23-year-old male with profound unilateral hearing loss for more than 10 years without a definitive diagnosis. In October 2016, a 23-year-old male patient came to CRG Hospital ENT OPD with chief complaint of decreased hearing in left ear for past 2 months. As per the patient, he was experiencing hearing loss from left ear for more than 10 years, but for the last two months the patient started having difficulty in hearing more from left ear than right ear, which was more profound when patient was using mobile phones or headphones. Patient has no complaints of tinnitus, headache or discharge from B/L ears, vomiting, vertigo, chronic systemic illness like DM, HTN or asthma.

Patient gives no similar family history. On physical examination patient was awake, conscious and well oriented to time, place and person with normal blood pressure and pulse with no icterus, pallor, cyanosis, clubbing, cervical lymphadenopathy, organomegaly or oedema. On ear examination, bilateral ears had normal external auditory canal with intact and normal tympanic membranes.

Examination of the nasal cavity revealed deviated nasal septum towards right side. Throat examination was normal with normal vocal cords on indirect laryngoscopy. On examination by tuning fork tests, no perception of sound is noted in the left ear on absolute bone conduction test and Rinne's test; however, Weber is lateralised to the right ear.

\section{PATHOLOGICAL DISCUSSION}

Cochlear aplasia or complete absence of the cochlea is most likely due to arrested development of the inner ear in the later part of the 3rd week of gestation or as described by some in the 5 th week. It is a rare anomaly accounting for only $3 \%$ of cochlear malformations. ${ }^{1}$ In cochlear aplasia the vestibule and semicircular canals are often malformed but may be normal. ${ }^{2}$ Dense otic bone is present at the site where the cochlea normally would be and is best depicted on CT images. It is important to differentiate this anomaly from labyrinthitis ossificans, in which normalised bone is seen anterior to the IAC with the bulge of the cochlear promontory produced by the basal turn of the cochlea, both features are absent in cochlear aplasia.

'Financial or Other Competing Interest': None.

Submission 12-10-2017, Peer Review 25-11-2017,

Acceptance 01-12-2017, Published 23-12-2017.

Corresponding Author:

Rajesh Kumar,

Flat no 88,

Amba Enclave, Sector 9

Rohini, Delhi-110085.

E-mail: tokasrajesh@gmail.com

DOI: $10.14260 /$ jemds $/ 2017 / 1533$
We report one such rare case of a 23-year-old male who had unilateral profound hearing loss for more than 10 years and has been investigated several times, but no definitive diagnosis was made. Physical and ear examination were normal and tuning fork tests and audiogram show sensorineural type of unilateral hearing loss. ${ }^{2}$ When the patient was further investigated, CT temporal revealed that normal cochlea and vestibular apparatus was not identifiable and dense bone is present at the location of cochlea suggesting the diagnosis of cochlear aplasia.

Cochlear aplasia is defined as the total absence of the cochlea with a present, although malformed vestibule. In this deformity the cochlea is completely absent, presumably as a result of an arrest in the development of the cochlear bud at the fifth week. This morphologic pattern is rare. Relative incidence of cochlear malformations are incomplete partition (Mondini) 55\%, common cavity 26\%, cochlear hypoplasia $15 \%$, cochlear aplasia 3\%, complete labyrinthine aplasia (Michel) 1\%. Ears with cochlear aplasia are devoid of auditory function and suffer from profound unilateral sensorineural hearing loss. ${ }^{2}$ Such patients suffering from profound unilateral hearing loss report no comprehension difficulty in a quiet one-on-one conversation; however, understanding conversation in less than ideal listening situations is severely compromised. These include noisy areas such as restaurants, group conversations and reverberant areas such as temples, gyms and indoor swimming pools. On examination by tuning fork tests, no perception of sound is noted in the affected ear on absolute bone conduction test and Rinne's test; however, Weber is lateralised to the better ear. Radiological features ${ }^{3,4}$ includes complete absence of the cochlea, dense otic bone at the anatomical site of the cochlea, absent cochlear nerve canal and cochlear nerve, hypoplastic and flattened cochlear promontory, vestibule and semicircular canals are often malformed, stunted, dilated but may be normal. Vestibular aqueduct is normal, internal auditory canal can be hypoplastic, facial nerve canal usually is anomalous showing obtuse angle anterior genu, middle ear is usually normal sized with normal ossicles. Imaging differentials include labyrinthitis ossificans, common cavity deformity, cystic cochleovestibular anomaly ${ }^{1}$ and labyrinthine aplasia. Treatment modalities include cochlear implant, auditory brainstem implant and bone-anchored hearing aid (BAHA).

\section{DISCUSSION OF MANAGEMENT}

Finally coming to our case, 23-year-old patient with singlesided SNHL-BAHA was advised. Patient was counselled of the pros and cons of the implant and after some consultation with his relatives, patient refused for any kind of surgical procedure. So, finally we advised the patient to take care of the only good hearing ear. 


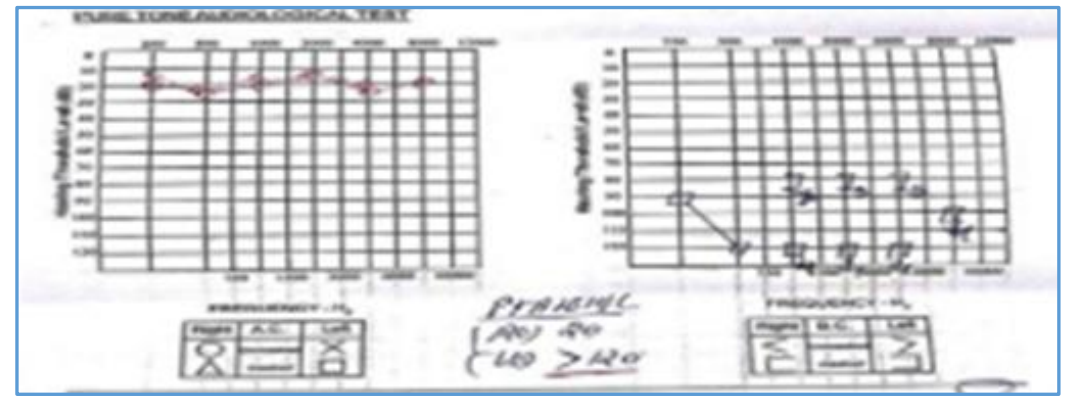

Figure 1. Pure Tone Audiogram showing Left Ear Sensorineural Hearing Loss

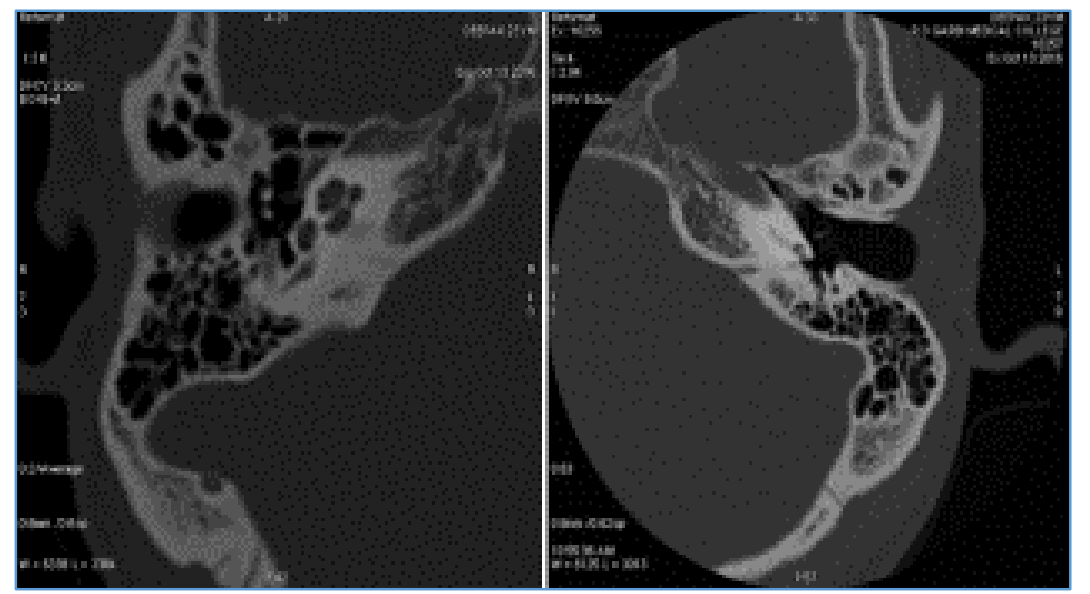

Figure 2 and 3. Axial Images of CT Temporal Bone shows Normal Cochlear Turns of Right Side and Dense Otic Bone at the Location of Cochlea on Left Side
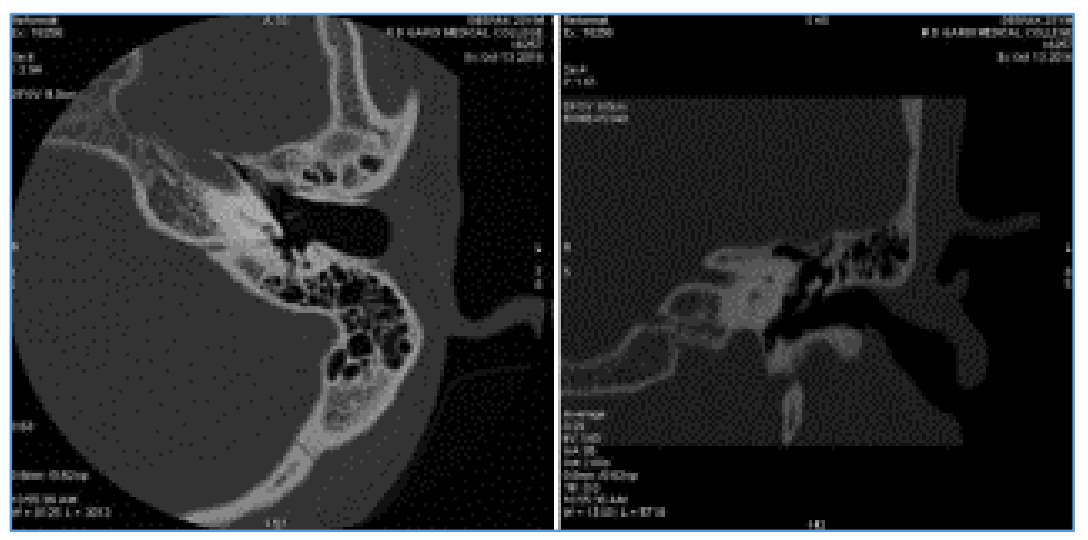

Figure 4 and 5. Shows Normal Ear Ossicles, however Normal Vestibular Apparatus is Not Identifiable, along with a Dense Bone at the Site of Cochlea in Axial and Coronal Planes

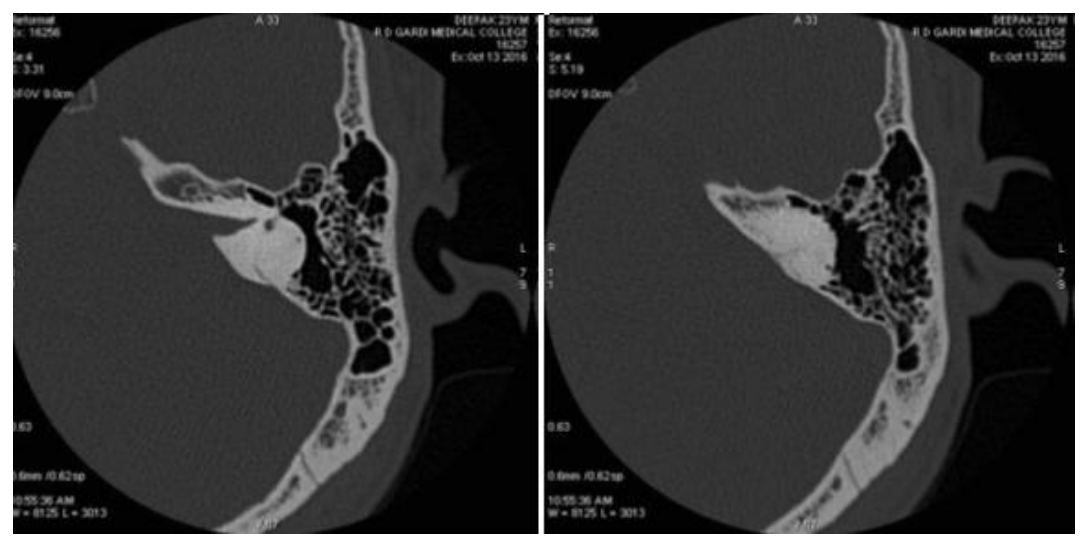

Figure 6 and 7. Axial CT Left Temporal Bone Images showing Dense Otic Bone 


\section{REFERENCES}

[1] Papsin BC. Cochlear implantation in children with anomalous cochleovestibular anatomy. Laryngoscope 2005;115(1 Pt 2 Suppl 106):1-26.

[2] Song JJ, Choi HG, Oh SH, et al. Unilateral sensorineural hearing loss in children: the importance of temporal bone computed tomography and audiometric followup. Otol Neurotol 2009;30(5):604-8.
[3] Joshi VM, DNB, Navlekar SK, et al. Neurologic/head and neck imaging. CT and MR imaging of the inner ear and brain in children with congenital sensorineural hearing loss. Radio Graphics 2012;32(3).

[4] Yiin RS, Tang PH, Tan TY. Review of congenital inner ear abnormalities on CT temporal bone. Br J Radiol 2011;84(1005):859-63. 